Palimpsesto Vol. 10, № 17 (enero-junio, 2020): 50-61

Universidad de Santiago de Chile, ISSN 0718-5898

María Luz Gómez Groppa

Universidad Nacional de Cuyo/Agencia Nacional de Promoción Científica y Tecnológica

marialuzgroppa@gmail.com

\title{
Redes para la resistencia: Puntos de contacto entre Argentina y Chile a través de las revistas político-culturales*
}

\section{Nets for the Resistance: Contact Points between Argentina and Chile through Political-Cultural Magazines}

\begin{abstract}
Resumen
El presente trabajo indaga el rol de las revistas político-culturales Claves para la interpretación de los hechos y Alternativa Latinoamericana, publicadas en Mendoza (la primera entre 1970 y 1974, y la segunda entre 1985 y 1990), en la configuración de redes para la denuncia de las violaciones a los derechos humanos que llevaban adelante las dictaduras cívico-militares en los países del Cono Sur, como así también la difusión de ideas en torno a los procesos de transición y retorno a la democracia, y a las alternativas de futuro para América Latina. Focaliza las relaciones entre Argentina y Chile, y pone el acento en aquellos artículos que, siendo censurados y/o difundidos de forma clandestina o subterránea en su país, encontraron en estas revistas un espacio garante de la circulación de ideas sobre la situación político-social chilena a partir del Golpe de Estado a Salvador Allende y de las denuncias a la dictadura encabezada por Augusto Pinochet.
\end{abstract}

Palabras claves: revistas político-culturales, Latinoamérica, dictadura, redes.

\begin{abstract}
This paper investigates the role of the political-cultural magazines Claves para la interpretación de los hechos and Alternativa Latinoamericana, published in Mendoza (the first between 1970 and 1974, and the second between 1985 and 1990), in the configuration of networks to denounce the human rights violations carried out by the civil-military dictatorships in the countries of the Southern Cone, as well the dissemination of ideas about the processes of transition and return to democracy, and the alternatives of the future for Latin America. It focuses on the relations between Argentina and Chile, and emphasizes those articles that, being censored and / or disseminated clandestinely or underground in their country,
\end{abstract}

* Este trabajo forma parte de la investigación del proyecto del PICT 2015-2019 "La historiografía de la cultura literaria latinoamericana a través de las redes intelectuales" coordinado por el Dr. Claudio Maíz (CONICETUNCUYO) financiado por la Foncyt de la Argentina. 
found in these journals a space that guarantees the circulation of ideas about the political-social situation Chilean from the coup d'état to Salvador Allende and the denunciations to the dictatorship headed by Augusto Pinochet.

Keywords: political-cultural journals, Latin America, dictatorship, networks.

\section{Puntos de contacto}

La reflexión en torno al pasado reciente en la historia de los pueblos latinoamericanos encuentra puntos de contacto e interrelación que, más allá de las particularidades y diferencias (o además de ellas), nos permiten pensar en América Latina como un territorio en el que se entrecruzan o confluyen trayectorias comunes. A partir de esto, si ponemos nuestra mirada en el pasado dictatorial de los países del Cono Sur, podemos identificar algunos rasgos que, según Elizabeth Jelin $(2002,2017)$, caracterizan la vinculación de estos países a una región que, además de territorial, es política. Entre los puntos de contacto, la autora reconoce: en primer lugar, una historia de fronteras porosas que incluyen movimientos y exilios políticos, contactos, vínculos, alianzas y lazos de solidaridad; segundo, el accionar coordinado y regional de la represión en los procesos dictatoriales latinoamericanos; como contrapunto, en tercer lugar, la conformación de redes de solidaridad y denuncia de las violencias a los derechos humanos, intercomunicadas e integradas de forma global y activamente regional; cuarto, las interrelaciones, puntos de interdependencia y trayectorias comunes (no autónomas y paralelas) en los procesos de transición en estos países durante las décadas del 80 y 90; por último, en quinto lugar, destaca cómo el pasado dictatorial reciente no quedó clausurado en el momento de la transición política y continúa siendo tema central del escenario político regional.

Los trazos visualizados por Jelin son claves para el análisis de aquellas redes que se articularon entre Chile y Argentina (durante y luego de los procesos militares inaugurados a partir de 1973 y 1976) como forma de resistencia a las dictaduras en los países del Cono Sur y como espacios para la denuncia de las violaciones a los derechos humanos por parte de las mismas y la divulgación de ideas en torno a los procesos de transición democrática y a las alternativas de futuro para la región. Redes que actuaron en el plano de las vinculaciones político-sociales en cuanto, por ejemplo, a los movimientos de exiliados ${ }^{1}$, pero también en el plano cultural a través de grupos de intelectuales, periodistas, artistas, escritores, que gestionaron diversos proyectos para garantizar la circulación de aquellas ideas que los regímenes militares censuraban o pretendían silenciar. Entre estas actividades, las revistas políticos-culturales tuvieron un rol activo

\footnotetext{
${ }^{1}$ En este punto, resulta pertinente mencionar las investigaciones de Alejandro Paredes (2007, 2013), quien comenta que el movimiento de exiliados chilenos durante las décadas del 70 y 80 en Mendoza, a partir del golpe de Estado al presidente Salvador Allende, fue el segundo más importante de la historia de ambos países. Según el autor: “...desde fines de 1973 a principios de 1975, ingresaron a Mendoza 107.800 chilenos. Casi siete mil obtuvieron el estatus de refugiados políticos por el Alto Comisionado de las Naciones Unidas para los refugiados (ACNUR) y fueron asistidos por el CEAS (Comité Ecuménico de Acción Social) con apoyo del Consejo Mundial de Iglesias. Después de la reapertura democrática argentina en 1983, hubo un acentuado crecimiento de la participación de los chilenos en organizaciones que presionaron para que su país se normalizara institucionalmente" (Paredes, 2013, p.53).
} 
importante que fue variando según los contextos políticos de cada país ${ }^{2}$ y que podríamos sistematizar de modo general en tres grandes etapas: de 1973, a partir del Golpe de Estado a Salvador Allende, hasta 1976, con el inicio de la dictadura en Argentina, surgieron diversas revistas encargadas de comunicar lo que sucedía en Chile y denunciar las atrocidades cometidas por el régimen pinochetista que ya comenzaban a conocerse y que encontraban en el país vecino espacios de difusión y repudio. De 1976, con el inicio de la dictadura en Argentina, y hasta 1983, momento del retorno a la democracia en este país, desatados ya los golpes cívico-militares en ambos países y con el Plan Cóndor en plena vigencia, las posibilidades de denuncia o análisis crítico de la realidad se vieron frustradas, muchas revistas desaparecieron y solo muy pocas fueron las que de modo "subterráneo" (Badenes, 2017) y clandestinamente pudieron referirse, cuestionar o denunciar los Golpes de Estado. Por último, de 1983 a 1999, finalizada la dictadura Argentina y hasta el inicio del proceso de transición democrática en Chile, surgieron revistas políticos-culturales que buscaron rearticular las redes de intelectuales que habían sido destruidas o silenciadas por los procesos militares, promover un espacio de reflexión en torno a sus consecuencias, retomar el debate frustrado acerca de la situación de América Latina y las posibilidades de liberación o transformación, y garantizar la difusión internacional de las denuncias de los crímenes cometidos por las dictaduras, sus atrocidades y sus continuidades.

En este contexto, en la provincia de Mendoza (ciudad argentina que por poseer uno de los cruces fronterizos más próximos a la capital chilena fue central en la relación entre estos países) aparecen dos revistas político-culturales con un rol activo en la configuración de redes de resistencia en Argentina y Chile: Claves para la interpretación de los hechos y Alternativa Latinoamericana. La primera se publica de forma quincenal entre junio de 1970 y diciembre de 1974, financiada por apoyos publicitarios privados y gubernamentales, y las suscripciones y ventas en kioscos. Es dirigida por Fabián Calle ${ }^{3}$ y cuenta con la participación de Miguel Longo, Norma Sibilla, David Eisenchlas, Arnaldo Cocola, Dante Di lorenzo, Carlos Quiros, Dante Polimeni, Raúl Fain-Binda, entre otros y otras. Al mencionar sus objetivos, se proponen participar del debate político tanto provincial como nacional e internacional ${ }^{4}$, interesados por aquellas noticias "claves" a menudo ignoradas, y declaran: "No haremos periodismo de escritorio. No esperaremos comunicados de prensa. Recogeremos opiniones [...]. Provocaremos respuestas sobre hechos y sucesos inmediatos. Los interpretaremos. Haremos prospectiva" (Claves: 1970, $\mathrm{N}^{\circ} 1, \mathrm{p} .1$ ).

\footnotetext{
${ }^{2}$ Para profundizar el desarrollo de las revistas político-culturales en Argentina son importantes las investigaciones realizadas en este campo por Noé Jitrik, Beatriz Sarlo, Daniel Badenes. Para el caso de Chile, recomendamos los trabajos de Horacio Eloy (2000) y Alexis Figueroa.

${ }^{3}$ En torno a la figura de Fabián Calle, como explica González (2011), interesa destacar que "provenía de la familia propietaria del medio gráfico histórico de la provincia de Mendoza, como lo fue y sigue siendo el diario Los Andes. Los destinos de los descendientes del fundador de la publicación siempre se habían mantenido relacionados con el propio diario, aunque Fabián, por diferencias intelectuales y personales con Felipe Calle (su tío y autoridad en el matutino) decide transitar otros caminos en materia periodística. Es así que junto a otros colegas coinciden en la decisión de fundar una nueva publicación que aborde temas políticos, sociales, económicos y culturales, dándole paso a revista Claves" (González, 2011, p.52).

${ }^{4}$ Son escasos los estudios en torno a la revista "Claves" por lo que su investigación y análisis es un campo a desarrollar. Entre los más conocidos, puede mencionarse la breve descripción que de ella realiza Gloria Rivera de Videla (2000) en su libro Revistas culturales de Mendoza y, de forma más completa pero abocada a un período específico de la misma, la investigación de Alfredo J. González (2011) "Juicio político al gobernador Alberto Martínez Baca: la intencionalidad editorial asumida por revista Claves y las ediciones dominicales de diario Los Andes".
} 
En cuanto a la segunda revista, Alternativa Latinoamericana surge en 1985 y se publica hasta 1990, durante cinco años y con once números, financiada por la organización Acción Popular Ecuménica (A.P.E.) ${ }^{5}$. Editada bajo la dirección de Rolando Concatti, cuenta con el diseño de Eduardo Williams Hermes Ruccio (desde la provincia de Córdoba) ${ }^{6}$, y la participación de diversos pensadores y pensadoras como Oscar Bracelis, Ricardo Rojo, Carlos Sota, Arturo Roig, Alberto Parisí, Osvaldo Ardiles, Norma Fernandez, Erio Vaudagna, Pedro Avejera, Julio Ruiz, Aníbal Sicardi, entre otros y otras. En sus objetivos, desde el primer número, se declara el interés por reconstruir las redes de diálogo y pensamiento que las dictaduras quebraron:

Queremos escuchar y queremos difundir. Romper el enclaustramiento. Volver a escuchar algunas viejas palabras que casi se nos habían olvidado; y escuchar otras nuevas que no conocíamos y que circulan, como una profecía, una levadura, una sabiduría también, por todo el Nuevo Mundo. (Alternativa Latinoamericana, $\mathrm{N}^{\circ} 1,1985$, p.5)

A partir del estudio de estas revistas, este trabajo propone una aproximación a Claves para la interpretación de los hechos y Alternativa Latinoamericana, las cuales, pese a su alcance e importancia en las épocas mencionadas, poco se han investigado y se presentan hoy como elementos importantes para la comprensión de los entrecruzamientos y diálogos entre los países latinoamericanos en nuestro pasado reciente. El acercamiento propuesto tiene como objetivo visualizar las redes que a través de estas revistas se establecieron entre Argentina y Chile para la denuncia de las violaciones a los derechos humanos que llevaban adelante las dictaduras cívicomilitares en los países del Cono Sur, como así también la divulgación de ideas en relación con los procesos de transición y retorno a la democracia. Para ello, indaga en aquellos artículos que, siendo censurados y/o difundidos de forma clandestina o subterránea en su país, encontraron en estas revistas mendocinas un espacio garante de la circulación de un pensamiento crítico en torno la situación político-social chilena a partir del Golpe de Estado a Salvador Allende y de las denuncias a la dictadura encabezada por Pinochet.

${ }^{5}$ Acción Social Ecuménica surge como una red social de nivel nacional en 1972 vinculada al Movimiento de Sacerdotes para el Tercer Mundo en la que participan Rolando Concatti, Oscar Bracelis, Alieda Verhoeven, Mauricio López, entre otros. En la nota editorial del primer número de Alternativa Latinoamericana de 1985, la A.P.E. definida por ellos como "una organización pluralista, orientada a generar proyectos y servicios de promoción social, de inspiración cristiana, abierta a quienes quieran participar en el proceso de liberación". Es importante destacar que, en articulación con esta organización, surge en Mendoza la Asociación Ecuménica de Cuyo y el Movimiento Ecuménico por los Derechos Humanos, organismos que junto al Comité Ecúmenico de Acción Social, llevan adelante una intensa actividad de solidaridad y ayuda con los exiliados y las víctimas de la dictadura chilena. Para profundizar el estudio de esta red de instituciones, sugerimos la lectura de las investigaciones de Alejandro Paredes "Santiago de Chile y Mendoza, Argentina: La red social que apoyó a exiliados chilenos (1973-1976)" (2016) y "El exilio no fue dorado... Las condiciones de vida de los exiliados chilenos en Mendoza entre 1973 y 1989"

${ }^{6}$ Como expresa Barón Del Pópolo (2018), la edición de la revista Alternativa Latinoamericana mantenía ciertas similitudes con la revista Crisis y una de las razones de esto era el compartir su diseñador: "Tenía una edición muy cuidada, otra de las características en común con Crisis. De hecho, compartían al mismo diseñador gráfico: Eduardo Williams Hermes Ruccio, mejor conocido en el mundo de la prensa gráfica como «Sarlanga», lo cual explica la similitud en las tapas y la diagramación entre una revista y otra". 


\section{Claves para la interpretación de los hechos}

"CLAVE -legisla la Academia de la Lengua- es una noticia o idea por la que se hace comprensible algo que era enigmático" (Claves, $\left.\mathrm{N}^{\circ} 1,1970, \mathrm{p} .1\right)$. Con estas palabras comienza la editorial que, bajo el título "A los lectores", introduce el primer número de la revista del 26 de junio de 1970 y explica la vinculación del nombre de la misma con su objetivo: la propuesta es intercambiar información y estudiar junto a sus lectores y colaboradores las "claves" del momento histórico. De esta manera, Claves para la interpretación de los hechos surge con la intención de promover el análisis político, económico, social, cultural y se presenta a un público que quiera dialogar con ella por lo que acompaña su título de tapa con una leyenda que anticipa: "La revista de Mendoza para la gente que disiente y discute".

Si bien son diversos los ejes de trabajo y temáticas que aborda en cada número (Gobierno, Política, Economía, Universidad, El mundo, Gente, Deportes, Vida moderna, son algunas de sus columnas), el debate político local y nacional articula el proyecto editorial (Olguín, 2013; Guevara, 2013). Ya en su número 100 de 1974, quienes realizan la revista explican:

CLAVES nació, por el sistema de suscripción, para denunciar los atropellos de la dictadura militar contra el pueblo; nació para canalizar la actividad política -no obstante la proscripción que pesaba sobre todos los partidos- que, subterráneamente desplegaron los distintos sectores. (Claves, 1974, p.6)

De esta manera, en un claro posicionamiento frente a la dictadura argentina de Onganía, reclama la necesidad de espacios donde el debate en torno a la acción política pueda darse libremente, sin censuras ni de forma clandestina. Tal vez es por este perfil que, pese a no tener mucho lugar la política internacional en sus primeros números, previo al Golpe de Estado a Salvador Allende y una vez iniciada la dictadura de Augusto Pinochet en septiembre de 1973, son frecuentes y reiteradas las noticias de análisis internacional de la situación político-social en Chile como así también las denuncias de la represión ilegal, torturas, secuestros y asesinatos cometidos por el régimen pinochetista.

\section{Chile: el "terror blanco"}

En septiembre de 1973, Claves anunciaba lo sucedido con Salvador Allende en Chile. A partir de esta fecha, las noticias en referencia a la dictadura de Augusto Pinochet son frecuentes y abarcan tanto los análisis políticos, los debates con el cónsul chileno en Mendoza, las producciones literarias o artísticas, como los testimonios de víctimas, las denuncias de las violaciones a los derechos humanos, las investigaciones de periodistas chilenos censuradas en su país.

A poco más de un mes de iniciado el Golpe en Chile, en octubre de 1973, con una nota titulada "Chile: el terror blanco", acompañada de un copete que declaraba: "Hasta ahora fusilamiento y torturas son los "argumentos" de la Junta Militar chilena" (Claves, N 81, 1973, p.48), la revista asume un fuerte compromiso por difundir lo que sucede en el país vecino: 
Mientras tanto en Chile. Tras la cordillera, las ejecuciones continúan. En las callampas, las poblaciones marginales, cien por cien simpatizantes de Allende, aparecen todas las madrugadas cadáveres de obreros, eliminados en la noche, al amparo de la impunidad que los uniformados tienen por el toque de queda. Se ejerce el "terror blanco" (por oposición a lo que sería el "terror rojo" de un gobierno popular que no mató a nadie, que no vejó ni torturó. (Claves Año IV, Nº 81, 1973, p.51)

Además de las noticias informativas y de los análisis políticos, quienes realizan la revista se encargan de investigar y hacer público los testimonios de las víctimas de la dictadura con notas como por ejemplo: "Relato de un mendocino que estuvo preso en Santiago, y que ahora no quiere olvidar. Chile: Testimonio sobre torturas". En la misma entrevistan a Carlos Rubén Espinosa quien, luego de haber estado preso en el Estadio Nacional de Santiago y de haber sufrido torturas, decide hacer pública su denuncia y exponer la represión que se vivía en Chile con la intención de movilizar al repudio para terminar con la dictadura: "Lo importante es presionar, demostrar que se está atento, que se siguen de cerca los acontecimientos. Hay que peticionar a la Junta Militar, formar comités (de barrio, de cualquier cosa) y parar esto. Hay que hacer todo lo posible" (Claves, $\mathrm{N}^{\circ} 81,1973$, pp.50-51).

Así, las referencias a la situación del país trasandino son continuas. Notas como "Chile: operación masacre. Para la Junta Militar chilena la prensa mundial es comunista y Kennedy un "tonto útil" (Claves, № 82, 1973, p.27), "Testimonio: Chile en vivo y en directo. Un grupo de jóvenes mendocinos sabe de primera mano cómo maneja su país la Junta Militar de Chile" (Claves, $\mathrm{N}^{\circ} 88,1974$, pp.38-39), muestran el hilo y tono que en cada número se entreteje. De hecho, desde septiembre de 1973 hasta el cierre de la revista, solo un número no tuvo alguna noticia que refiriera a Chile, situación que ocasionó el cuestionamiento de sus lectores y la respuesta automática: "¿Por qué se han callado respecto de Chile? La pregunta, hecha telefónica, postal y personalmente a los miembros del staff de CLAVES, revela la necesidad de información que tiene el mendocino sobre lo que sucede en el resto del mundo" (Claves, $\mathrm{N}^{\circ}$ marzo 1974, p.47).

En relación con el reconocimiento de una necesidad de información por parte de los lectores, resulta interesante destacar el esfuerzo por publicar noticias sobre Chile de diversas fuentes. Así, en febrero de 1974, Claves publica un número en el que recopila material acerca de la dictadura pinochetista distribuido por distintas agencias norteamericanas (UPI y AP), alemanas (Reuter), francesas (France Press), latinoamericanas (Latin, Telam, NA), y editado por varios diarios como The New York Times, Washington Post, Le monde, La opinión, Clarín, Noticias, etc. Esta publicación va acompañada de una nota aclaratoria que resulta pertinente para entender el alcance de la tarea que la revista se proponía:

CLAVES publicita estas fuentes de información con la finalidad especial de que el mendocino comprenda hasta qué punto vive periodísticamente "aislado" de un país del que lo separa apenas una frontera de piedras. A poco más de $200 \mathrm{~km}$ de Santiago de Chile, Mendoza debe recurrir a las noticias que provienen del Atlántico para enterarse de lo que sucede en el Pacífico. (Claves, № 88, 1974, p.36)

Como ya hemos mencionado, las redes de resistencia a las dictaduras militares, intercomunicadas e integradas de forma global y activamente regional, fueron fundamentales para su denuncia. Quienes por la represión no podían denunciar las torturas y violencias sufridas 
en su país, encontraban en Argentina posibilidades de hacerlo. En este sentido, Claves brindó el espacio para que chilenos pudieran expresar y publicar sus palabras. Así, por ejemplo, en septiembre de 1974, a un año del Golpe de Estado, aparece "CHILE: Otros cinco asesinatos", una investigación de un grupo de periodistas clandestinos que desmontaba el supuesto descubierto Plan Leopardo por parte del gobierno militar en el que se informaba de la muerte de cinco "extremistas" y con el cual se encubría el asesinato brutal de cinco personas por parte de las fuerzas militares: "Un grupo de "periodistas clandestinos" de Chile decidió investigar los hechos por su cuenta y después de varios meses logró elaborar su propio informe. Después de muchos avatares, el mismo llegó a CLAVES. Aquí está" (Claves, № 100, 1974: 23).

Con un fuerte posicionamiento en contra de las violaciones a los derechos humanos en Chile y la defensa de la democracia, alertando lo que podría suceder en Argentina, en un contexto de fuertes cuestionamientos políticos, Claves para la interpretación de los hechos deja de publicarse, como explica González (2011), hacia fines de 1974 luego de sufrir un atentado en la imprenta en la que se confecciona la revista (el cual luego es atribuido a la Triple A).

\section{Alternativa Latinoamericana}

Con una cita del ensayo "Nuestra América" que el pensador cubano José Martí publica en 1891 y una ilustración del artista mexicano José Guadalupe Posada, pertenecientes al libro "Posada y las calaveras vivientes" publicado en 1979, se introduce el primer número de Alternativa Latinoamerica evidenciando el carácter histórico del diálogo que pretenden retomar, de las voces que quieren volver a escuchar, quienes realizan esta revista. En la nota editorial que sigue a esta introducción, los mismos contextualizan, ponen en situación a sus lectores y lectoras, y refieren sus objetivos e intenciones. Es claro y evidente que, retomada la democracia en Argentina en 1983, las secuelas y consecuencias de la dictadura cívico-militar deben ser enunciadas y el enclaustramiento, la censura y los silencios impuestos por los procesos militares deben transformarse. Silencios determinados no solo por la imposibilidad de expresar las ideas, de hablar, sino también por la imposibilidad de escuchar, es decir, por la ruptura en las redes de diálogo y el acallamiento las voces:

Hemos vivido en el silencio, pero no solo porque no podíamos hablar, sino porque tampoco podíamos escuchar. Los jóvenes sobre todo, pero también los mayores, hemos vivido esta etapa sin esa red de relaciones horizontales que lo hacen a cada uno contemporáneo de su tiempo, que lo hacen vivir la hora de su historia. Muchos tenemos la convicción, o el presentimiento de que esa historia pasa hoy, medularmente, por América Latina. (Alternativa Latinoamericana, $\mathrm{N}^{\circ} 1,1985, \mathrm{p} .5$ )

Así, la publicación de una revista en su tiempo aparece como una forma de retomar la palabra, superar las experiencias solitarias, multiplicar y avanzar en el diálogo, y reactivar o reconstruir una red de relaciones que posibilite el intercambio y la controversia con el objetivo declarado de "escuchar y difundir" ideas. En una entrevista realizada a Rolando Concatti por Guillermo Barón Del Pópolo (2016) acerca de la producción de esta revista, el mismo refiere a la pregunta que movilizó el proyecto: 
¿cómo nosotros no tenemos una revista que fije posiciones? Sobre dos o tres temas claros en América Latina y acá en la Argentina, de los que no se hablan, que están más o menos prohibidos, que sea suficientemente crítica de todo lo que pasó, pero que sea esencialmente positiva, prometedora de un futuro distinto (Barón Del Pópolo, 2016, p.150)

En este punto, la opción por Latinoamérica resulta central. El objetivo es recuperar los debates en torno a la liberación y unidad de sus pueblos, y las posibilidades de alternativas para su futuro, las líneas de trabajo interrumpidas y las redes de organizaciones e intelectuales de un pensamiento propio, local, quebradas por las dictaduras. Por esta razón, como expresa Barón del Pópolo $(2016,2018)$, en la revista confluyen diversas corrientes intelectuales latinoamericanas que habían sido censuradas y perseguidas como "la Filosofía de la Liberación (colaboraron en ella Arturo Roig, Enrique Dussel, Osvaldo Ardiles, Horacio Cerutti), el peronismo de la Renovación (Salvador Ferla, Hugo Chumbita), el viejo grupo de las Cátedras Nacionales y la Antropología 3er Mundo (Guillermo Gutiérrez, Horacio González, Aníbal Ford, Alcira Argumedo)" (2016, p.147).

En diálogo con estas corrientes, las temáticas abordadas por la revista abarcaron diversos ejes: la pedagogía latinoamericana y la educación popular (con análisis de Oscar Bracelis, Maria Ines Abrile de Vollmer), la teología de la liberación (con aportes de Raúl Vidales, Hugo Assmann, José Miguez Bonino, Floreal Forni, Javier Gorostiaga), las problemáticas económicosociales abordadas y los movimientos políticos y sociales (Barón Del Pópolo, 2016). A esta diversidad de temas, agregaremos: el feminismo latinoamericano (con aportes de Alieda Verhoeven, Domitila Chungara, Sofía Vega), los estudios culturales (con colaboraciones de Néstor García Canclini, Jesús Martín Barbeiro, Héctor Tizón), los estudios literarios (Roberto Fernández Retamar, Mario Benedetti, Eduardo Galeano, Juan Draghi Lucero, Horacio González), entre otros. Como puede reconocerse, este panorama de autoras y autores, de temáticas presentadas para el debate, de formas de abordaje desde una teoría crítica, se presentan en sintonía con lo opción enunciada en la editorial del primer número de la revista: "Lo importante está en que desde el principio ponemos a Latinoamérica como un eje decisivo, y no como mero contorno, como una referencia fina y no de algún modo exterior" (Alternativa Latinoamericana, $\left.\mathrm{N}^{\circ} 1,1985, \mathrm{p} .5\right)$.

La opción por América Latina va de la mano del posicionamiento político e ideológico: quienes realizan este proyecto afirman estar comprometidos con el proceso de liberación y la unidad del pueblo latinoamericano y consideran que una revista puede servir en esta tarea por lo cual la misma está dirigida "para" la militancia, para aquellos que, desde horizontes ideológicos distintos y prácticas concretas diversas, "son a la vez y prácticamente una conciencia y una voluntad de cambio"; y está hecha "desde" la militancia, realizada por un grupo que ha buscado y busca "el servicio de la causa popular" (Alternativa Latinoamericana, N¹, 1985, p.6). Si consideramos la trayectoria de quienes producen esta revista, varios provienen de experiencias de militancias durante los 60 y 70 en grupos de izquierda o peronistas, tanto de organizaciones partidarias como sociales, religiosas, académicas, culturales con fuerte diálogo y articulación latinoamericana. "Somos un grupo de viejos buscadores que hoy nos encontramos, después de la diáspora y el exilio de muchos, ateridos de preguntas pero fuertes en ciertas convicciones" (Alternativa Latinoamericana, $\mathrm{N}^{\circ} 1,1985, \mathrm{p} .6$ ), comentan. Y, en este encuentro que la reapertura democrática en Argentina posibilita, se entretejen las reflexiones en torno a las dictaduras que en otros países vecinos continúan. Así, la articulación con espacios que denuncien la dictadura en 
Chile y que promuevan el retorno a la democracia es una tarea asumida como militante, por lo cual la publicación de notas que refieran a la situación del país vecino y que intervengan en la modificación de su realidad tendrá un espacio en la revista.

\section{Chile Hoy. "1986: Seamos realistas, nunca es imposible"}

Como explica Barón Del Pópolo, la reproducción de artículos de diversas revistas de América Latina por parte de Alternativa Latinoamericana era frecuente. Por ejemplo, se publicaban artículos de la revista "Socialismo y Participación de Perú, la revista Pasos del DEI de Costa Rica, Tempo\&Presença de Brasil, entre otras" (2016, p.147). Pero en 1986, en el volumen número IV de la revista, se publica un artículo que merece nuestra atención. El mismo se titula "1986: seamos realistas, nunca es imposible" y aparece en una columna denominada "Chile Hoy" (Alternativa Latinoamericana, $\mathrm{N}^{\circ} 4$, 1986, pp.66-69). El artículo pertenece a la revista chilena $\mathrm{Krítica}^{7}$ (de la cual, en cuanto a los datos de edición, solo conocemos que surgió en mayo de 1978 y fue de aparición bimestral) que, según comentan los responsables de la publicación en Argentina, ha llegado a sus manos pese a las dificultades a enfrentar con la dictadura y su circulación restringida. Es importante la aclaración de este detalle ya que demuestra una acción concreta en relación con la ruptura del enclaustramiento y silenciamiento antes mencionado.

Esta revista, como ellos mismos dicen: "vio la luz en mayo de 1978 ocupando un espacio comunicativo sin pedirle permiso a nadie y porfiando con ideas que los hombres armados estaban haciendo lo posible por eliminar. Al compás de la reconstrucción del movimiento popular, del surgimiento de expresiones culturales y de los episodios de la vida y la lucha en el Chile dictatorial, fuimos haciendo un aporte -al principio como hoja mimeográfica- a la renovación política que todos necesitábamos para dejar atrás la derrota y buscar caminos de victoria. (Alternativa Latinoamericana, $\mathrm{N}^{\circ} 4,1986, \mathrm{p} .66$ )

Este artículo propone un análisis político extenso y detallado acerca de la situación del gobierno dictatorial de Pinochet a trece años de iniciado el Golpe y las posibilidades de una salida popular y democrática del mismo. Las referencias al rol de las fuerzas armadas en la represión, la debilitada legitimidad de la dictadura y las consecuentes movilizaciones sociales que lentamente comienzan a visibilizarse, promueven nuevas visiones del país, nuevas alternativas políticas que posibilitan pensar en una transición y recuperación de la democracia como en Argentina. Todo este análisis circula en Chile clandestinamente pero encuentra aquí un espacio para la publicación y difusión.

El artículo, a diferencia de los publicados por la revista Claves, no se detiene en el detalle de las denuncias o testimonios de las víctimas de la dictadura ya que su objetivo consiste en promover el debate en torno a las posibles salidas y clausura de esta etapa. La reflexión parte de aquello que, en tantos años de dictadura y pese las persecuciones, siempre se ha defendido y que hoy resulta fundamental para la reconstrucción:

\footnotetext{
${ }^{7}$ Revista de la cual no hemos conseguido ver sus números originales pese a la consulta e investigación realizada en diversas bibliotecas (entre ellas la Biblioteca Nacional de Chile) y con diversos especialistas en Chile. Comentamos esto con el afán de promover su búsqueda.
} 
Desde lo que nos es propio y hemos defendido en todos estos años: la experiencia de base; los gérmenes de poder que emergen en el pueblo; la renovación del pensamiento socialista; el enriquecimiento del debate político; la preocupación por la cultura como factor de construcción de un país; los procesos sociales latinoamericanos como parte de lo nuestro; el rescate de la historia chilena reciente y remota a partir de sus protagonistas reales; la comunicación sin censuras de experiencias y posiciones diversas en este Chile escindido, exiliado y por reconstruir; el viento fresco de las nuevas ideas y sensibilidades crecidas a la sombra de la dictadura y contra ella. (Alternativa Latinoamericana, $\mathrm{N}^{\circ} 4,1986, \mathrm{p} .66$ )

\section{Palabras finales}

Como hemos mencionado, los puntos de contacto entre los países del Cono Sur que atravesaron las dictaduras cívico-militares son varios y no solo refieren a las articulaciones que se dieron entre las fuerzas represivas de cada país, sino también a las redes de resistencia a las mismas. Estas últimas movilizaron lazos de solidaridad para la protección de las víctimas, la recuperación de la democracia, y la denuncia y repudio a las violaciones a los derechos humanos cometidos por los genocidas y represores.

Entre las diversas acciones que durante las dictaduras se llevaron adelante para construir las redes de resistencia, la difusión y circulación de ideas fue fundamental. Así, las revistas político-culturales, proyectos que disputan en el espacio público el conflicto presente (Sarlo, 1992) y que buscan configurar otras maneras de pensar la realidad, sirvieron como soporte para aquello que intentaba ser silenciado, para aquello que fue quebrantado y que, una vez finalizadas las dictaduras, requería ser reconstruido. En este sentido, las revistas mendocinas Claves para la interpretación de los hechos y Alternativa Latinoamericana (en distintas épocas y con diferentes propuestas) formaron parte de esas redes y colaboraron en su construcción asumiendo la tarea de compartir y garantizar la divulgación de un pensamiento crítico de la situación político-social chilena a partir del Golpe de Estado a Salvador Allende y las denuncias a la dictadura encabezada por Pinochet, como así también fortalecieron el debate en torno a los procesos de transición y recuperación de la democracia en Chile, y a las alternativas de futuro para esa América Latina que, más allá de las fronteras territoriales, posee una historia común que la unifica en una región política marcada por las luchas y resistencias populares. 


\section{Referencias Bibliográficas}

Badenes, D. (2017). "Notas para una historia de las revistas político-culturales”. En: Badenes, D. (et. al) (2017). Editar sin patrón: La experiencia política-profesional de las revistas culturales independientes. La Plata: Club Hem. Disponible en: http://www.memoria.fahce.unlp.edu.ar/libros/pm.662/pm.662.pdf

Barón Del Pópolo, G. (2016). "La intuición de una América Latina distinta. Entrevista a Rolando Concatti sobre la revista Alternativa Latinoamericana”. En: Pelícano, 2, 146-155. Recuperado de: http://revistas.bibdigital.uccor.edu.ar/index.php/pelicano/article/view/1128 . (2018). “¿Puede hablar el pueblo? Educación, cultura y religiosidad popular en la revista Alternativa Latinoamericana". En: Nuevo Mundo Mundos Nuevos, Cuestiones del tiempo presente, Puesto en línea el 05 octubre 2018. Recuperado de: http://journals.openedition.org/nuevomundo/72993; DOI 10.4000/nuevomundo.72993

Eloy, H. (2000). "Revistas y publicaciones literarias durante la dictadura (1973-1990)". En: Simpson 7 / Sociedad de Escritores de Chile. Santiago: La Sociedad, 1991- volúmenes, tomo 12, (semestre 2 2000), páginas 75-89

Jelin, E. (2002). Los trabajos de la memoria. Buenos Aires: Siglo Veintiuno Editores. . (2017) La lucha por el pasado: cómo construimos la memoria social. Bs.As.: Siglo XXI Editores.

González, A. J. (2011). Juicio político al gobernador Alberto Martínez Baca: la intencionalidad editorial asumida por revista Claves y las ediciones dominicales de diario Los Andes (Tesina de grado). Mendoza: Universidad Nacional de Cuyo. Facultad de Ciencias Políticas y Sociales. Disponible en: https://bdigital.uncu.edu.ar/6501.

Guevara, E. (2013). "Historia de una mujer Clave". En: Unidiversidad, 07 de junio 2013. Disponible en: http://www.unidiversidad.com.ar/historia-de-una-mujer-clave

Sarlo, B (1992). "Intelectuales y revistas: razones de una práctica". En: América: Cahiers du CRICCAL, $\mathrm{n}^{\circ} 9-10,1992$. Le discours culturel dans les revues latino-américaines, 19401970. 9-16

Olguin, P. (2013). "El peronismo mendocino de cara a las elecciones de 1973”. En: Quinto Sol, Vol. 17, No 1, enero-junio 2013. Disponible en: http://dx.doi.org/10.19137/qs.v17i1.596

Paredes, A. (2007). "Santiago de Chile y Mendoza, Argentina: la red social que apoyó a exiliados chilenos (1973-1976)". En: Redes. Revista hispana para el análisis de redes sociales. Vol. 13. Disponible en: https://www.raco.cat/index.php/Redes/article/view/76627 . (2013). "Campos de poder, exilio y participación política en el país de origen. Los exiliados chilenos en Mendoza (Argentina) y su participación en el escenario político chileno (1983-1989)". En: SudHistoria. Vol. 6, enero-junio. 51-80. . (2016). "El exilio no fue dorado... Las condiciones de vida de los exiliados chilenos en Mendoza entre 1973 y 1989”. En: III Jornadas de Trabajo Exilios Políticos del Cono Sur en el siglo XX. Agendas, problemas y perspectivas conceptuales. Santiago de Chile 9, 10 y 11 de noviembre de 2016. Disponible en: http://jornadasexilios.fahce.unlp.edu.ar/iii-jornadas/actas-2016/Paredes.pdf 
Revista Alternativa Latinoamericana. (1985-1990) Mendoza: Secretaría de Estudios de APE. Acción Popular Ecuménica. Disponible en: Movimiento Ecuménico por los Derechos Humanos, Regional Mendoza.

Revista Claves para interpretar los hechos. (1971-1974). Mendoza: Periodista Asociados Editores. Disponible en: Biblioteca Popular General San Martín, Mendoza.

Videla de Rivero, G. (2000). Revistas culturales de Mendoza. Mendoza: Universidad Nacional de Cuyo. EDIUNC. Disponible en: http://bdigital.uncu.edu.ar/11920. 